\title{
A Review on Various Techniques to Transform Traditional Farming to Precision Agriculture
}

\author{
Rana Gill , and Dr. Paras Chawla ${ }^{b}$ \\ A Assistant Professor at Chandigarh University, Mohali, India. \\ ${ }^{\mathrm{B}}$ Professor, ECE department at Chandigarh University, Mohali, India.
}

Article History: Received: 11 January 2021; Accepted: 27 February 2021; Published online: 5 April 2021

\begin{abstract}
The agricultural sector is of great importance to fulfill food resources need of the country. Precision Agriculture (PA) with Internet of Things and Wireless Sensor Network is a transformation from traditional farming to smart farming. Wireless sensor networks and Internet of Things are considered as drivers to develop system which can change agriculture sector from manual to automatic. Advancement in the technology have pushed the growth of precision agriculture to very large extent despite of several challenges faced in this area. System for precision agriculture relies on hardware components mainly wireless sensors which act as a source for gathering of real time data. Depending upon the real time date retrieved by sensors automation in agriculture is done by adopting decision-based system. With Precision agriculture productivity is optimized by maintaining sustaniability as crop receives what is acutual requirement on the basis of new techniques and software platforms. This review article includes Inernet of Things (IoT), Wireless Sensors, Wireless communication and challenges faced in this area.
\end{abstract}

Keywords: Precision Agriculture, Wireless Sensor Networks, Internet of Things

\section{Introduction}

India has $16 \%$ of world papulation but have $4 \%$ of world's fresh water resources out of which $80 \%$ is used for agriculture [1]. India is known as agricultural country as $80 \%$ of its rural papulation have agriculture as their source of livelihood [2]. Many states of India have their agriculture totally dependent on rainfall due to very less availability of freshwater. This shortage of freshwater supply demands paradigm shift to productivity per unit of water. In various economic surveys done in past five years it is indicated that adoption of advancement in technologies is required to make agriculture sector sustainable for small and marginal farmers. Climatic changes that are affecting country in various ways, are going to lower farmer's income further by $25 \%$. Three factors under climate change pointed are decline in average rainfall, rise in temperature and more dry days. India's 73.2 million hectares area is still unirrigated area and only rainfall dependent out of 141.4 million hectares net sown area. Economic Survey alerted and recommended the requirement for "dramatic" improvement in irrigation by involving new technologies [3]. In traditional farming irrigation is either done by manual observation of farmer or it is scheduled irrigation. Additionally, water overuse can cause water shortage, often occurs in areas of irrigation agriculture, and harms the environment. Present scenario demands novel technology for increasing the crop productivity, and a technology to reduce the water consumption for irrigation. This is clearly indicating a transformation of traditional farming to Precision agriculture. Precision Agriculture (PA) not only increases crop yield and revenues but also involves methods which are very environment friendly. Precision agriculture is a way of farming which is done by considering all the elements related to soil moisture and temperature, environment details like temperature, humidity and rate of evaporation measured by technology-based devices. Precision agriculture is done more precisely on the basis of what is required, when is required and where is required in the farm. Precision farming is a technique of farming that changes the use of pesticides and fertilizers to evaluate the variation in the growth conditions within the agriculture field. This way of practice is termed as Site Specific Management (SSM) [4]. This way it reduces the amount of chemicals released into the environment. Precision agriculture also helps in reducing the requirement of man power which further helps farmers to have more economic benefits. Advancement in Cloud Computing, Big Data Analytics, Artificial Intelligence and Internet of things are very helpful to attain Precision agriculture. This review paper is focused on Internet of things and various techniques used in this field to agriculture technology based.

\section{Precision Agriculture}

Precision agriculture is management of farming on basis of observation, measurement and decision-based system. First revolution of precision agriculture started with use machinery in place of manpower and farming with help of animals. This revolution happened in early 19s (1900-1930). Second wave started with green revolution which enhanced agriculture on the basis genetics which led to precise plantation. Presently, third wave of revolution is going on which came into implementation in 1980 with the advanced technologies in agriculture [5]. The main focus of the review article is to study various methods opted by different researchers in past five years for smart farming. Various technologies that are helping different researchers are Big Data Analytics, Cloud Computing, Internet of Things, Wireless sensor network, Artificial intelligence, Image processing using 
Computer vision and many more. These technologies are helping to transform traditional farming to precision agriculture at very fast pace. Various technologies used in precision agriculture are shown below in figure1 [6].

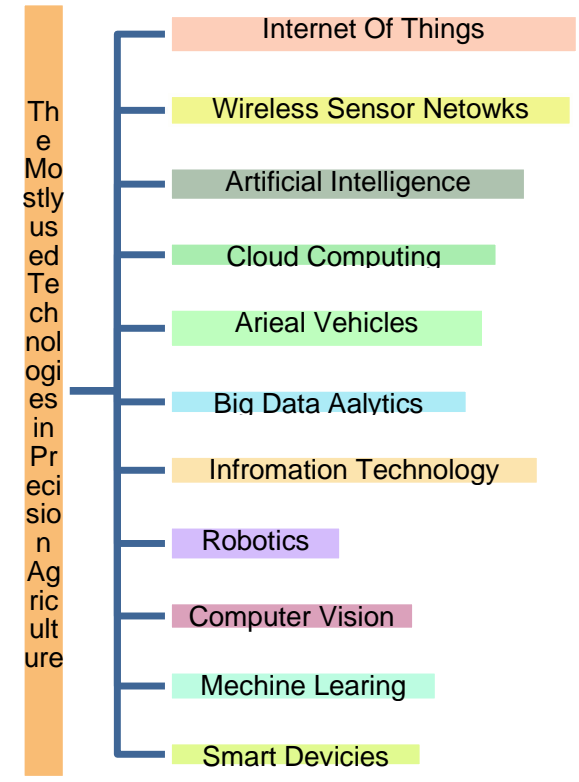

Fig. 1. Overview of Precision Agriculture Technologies

This article is framed to cover only those research article which Internet of things based. The survey presents the idea of Precision agriculture with various innovations from its cause, various technologies and their upgradations ideas. Precision Agriculture utilizes Internet of things (IoT) arrangements to increase productivity while reducing water consumption, manpower required and saving environment. In the early stages, Precision agriculture consisted mostly of map-based technologies using geo statistical methods such as GIS and satellite based remote sensing. And main focus was to apply to control fertilizer use. Earlier sensors were not commonly used because of high cost, unreliable or unavailable for necessary applications. Precision agriculture got started in its third wave during 1980s but till early 2000s not adopted by many farmers. The key obstacles to the adoption of such approaches were the lack of technology, lack of scientific evidence, no training and high cost [7]. Decreased sensors cost and rapid Internet of Things (IoT) and Big data growth lead to development and testing of more PA system. IoT works in form of cycle that firstly do monitoring with the help of sensor, analysis of information gathered and then control [8]. Further by involving Big data Analytics and machine learning decision making system can be designed [9].

\subsection{Internet of Things}

In agriculture the Internet of Things is about cameras, sensors and other smart devices to get data from every action in farm. Internet of things helps to build smart farming in various forms like sensing, watermanagement to increase crop yield, determine fertizer requirement, finding optimal time for planatation and harveting and weather conditions. IoT is a type of enviornment in which objects can transmit data over internet without or with minimal human interventation [10]. The IoT is designed around various wireless radio standards which are responsible for interaction of different devices with each other through the Internet. It comprises many standards like Zigbee, Bluetooth, LoWPAN, Wi-Fi ,NFC, Z WAVE, RFID, and so on. Physical object which collects and processes data that it receives about the enviornment [11]. These objects are built with sensors, actuators, microcontrollers and transceivers. The irrigation requirements can easily be automated by the IoT- driven decision support system (DSS). In [22] authors proposed system to transmit information through the internet with the help of internet of things The IoT allows devices to control remotely or sense physical environmental factors using sensor nodes. The user interface is generated with the help of an Android app. The data gathered by the sensor nodes propagates following tree topology to reach the base station. Arduino UNO and nodeMCU are used for transmission and soring the data collected to the cloud. Developed Android app enables the access of an information uploaded to the cloud.

Various technologies are growing under internet of things and many methods are proposed and implemented by various researchers in recent years.

\subsubsection{Wireless Sensor Network}

Wireless sensor Networks help in shift from traditional farming to smart farming. Research on Precision agriculture from basic climate information, soil information to decision support system for irrigation are dependent on collection of data by wireless sensors. The wireless sensor system not only improves crop productivity but also helps in saving environment in various forms [12]. A WSN usually consists of many multifunctional sensor nodes that are deployed in a interested field. With the recent advancements these nodes are of low cost, less power consumption and small in size. In spite of miniature structure these nodes are provisioned with sensors, microcontrollers, and radio transceivers. These nodes have capability to sense the data, process it and capable to communicate it. These nodes are capable to communicate over short distance via wireless mode 
of communication. Wireless communication standards plays an important role for normal functioning of wireless sensor network. Various techniques are designed at physical layer and protocols are followed at higher layers for effective working of wireless sensor network. Nodes are generally categorises as a source node and a sink or gateway node. The collection of data is done by source node and receiving of data is by sink or gateway node. In terms of computational power sink nodes have higher power in comparison to a source node. While choosing wireless nodes constraints faced are size, data rate, memory and power consumption. In [13] comparison of sensor nodes used in precision agriculture is done with their specification and it is considered that MICA2 is more suitable due to its capability to connect with many sensors with help of its large expansion connectors. In recent many ways are opted by different researchers to apply wireless sensor network to agriculture. In [14] method proposed to monitor agriculture environment was based on smart wireless sensor network. They design an architecture by deploying various nodes in the agriculture area. Then collected data was analysed and displaying it to end user. Similarly, In [15] authors proposed a technique which applies on top surface of the soil in two domains namely time and space to monitor variation in soil moisture content. This experiment has conducted by considering twenty seven different locations where wireless sensor nodes have deployed. To collect real-time data multi hope configuration has been used. In [16] paper has wireless sensor network based smart system used in a red bayberry greenhouse. A smart system is designed using temperature sensors and soil moisture. This system monitor the humidity ,temperature and illumination in the greenhouse. In [17] authors proposed wireless sensor networks based system in potato farm which monitors individual crop requirements. The farmers can easily recognise the different requirements of crop ,fertilizers use and need of irrigation. The article presented a model for irrigation management to figure out agricultural parameters using intelligent humidity sensors and mathematical calculations on the basis of received input. In [18] authors presented smart precision agriculture system using IoT technology and wireless sensor network. This system has designed automated irrigation system. Comparison of scheduled irrigation in which irrigation is done at specific times with automatic irrigation system based on moisture sensors, temperature, and humidity. Two irrigation methods are compared and it is found that automatic irrigation has better efficiency in terms of water consumption. In [19] proposed method is implemented by dividing the agricultural land in for regions and three different sensors namely temperature, humidity and soil moisture sensor. Data collection is done by these sensor nodes and transmitted through other nodes and gateways to server. Data received at server is analysed and displayed in an application in GUI that can be made available in mobile application and on the related website. In [20] authors developed IoT-enabled wireless sensor network environment to monitor soil and environmental parameters which are used to schedule irrigation on the basis of water requirements. Developed robust mechanism using interpolation technique for making soil moisture content distribution map. Method made distribution map by measuring soil moisture content at different nine positions of the agricultural land. Structural similarity index (SSIM) mechanisms was adopted to control irrigation valves depending on zone-specific irrigation control commands. In [21] a wireless drip irrigation system was presented by deploying soil moisture sensors. An implementation of a WSN was realized for wireless managed irrigation solution and real time monitoring of soil water content. Data collection is done with the help of solar powered based wireless acquisition stations to automate an irrigation. With this system design monitoring of water content was done. It is clear from above reviewed papers that proposed systems use multiple sensors nodes to make agriculture more precise. The design of the system is selected depending on various features that fulfil the requirement for low cost, less power consumption, less water consumption, reduced manpower and secure wireless communication between these nodes [23].

In the agriculture sector wireless sensor networks are being used to develop smart irrigation systems, smart fertilization systems, crop health monitoring, early crop disease detection and smart pest control systems.

\subsubsection{IoT Connectivity}

In IoT objects can talk and this is possible only through their connectivity to each other and to the outside world. Due to the rapid growth in WSN technologies and IoT devices several wireless communication protocols are brought in use. These protocols have their own specifications depending on various factors like data rate, bandwidth, free channels, , battery life and cost [24]. Commonly used protocol for IoT connectivity in agriculture sector are Zigbee, Bluetooth, 6LoWPAN, RFID, Wi-Fi and Lora WAN. ZigBee is a wireless protocol commonly used in precision agriculture applications like smart irrigation systems and smart greenhouses [25]. In [26], authors presented a smart irrigation system in which communication is handled by the ZigBee module. This system used two nodes, i.e., a sensor node and an actuator node where sensor node was comprised of soil moisture sensors, which were used to monitor the water level in the soil and the actuator module used to take required actions on basis of the water content of the soil. ZigBee protocol handled the whole communication part of the system. In [27] wireless greenhouse sensors based on CC2420 and Zigbee. In [28] greenhouse environment monitoring system using three sensors namely light intensity sensor, air temperature sensor and humidity sensor was designed. The system monitoring is Wi-Fi based which converts TTL signal from the sensor to the Wi-Fi signal to be transmitted to the server. In this framework physical devices are connected to Wi-Fi network to monitor and control greenhouse environment. Similarly, In another smart agriculture system proposed in [29] Wi-Fi protocols were used. In this paper system is designed using Raspberry Pi and multiple sensors used for data collection. The transmission of this data to the cloud is done using Wi-Fi protocols. Indu and S.R.N. [30] proposed a GSM(Global system for mobile communication) based system to control irrigation. The system designed is used to set the irrigation time depending on the data collected by temperature and humidity sensors which helps to irrigate automatically when unattended. Communication between the end user and system is 
established via short messaging service (SMS) on GSM network. In [31], a proposed system used smart phone using Bluetooth to collect the data from sensors deployed in the farm. Sensors used in the system are soil temperature sensor and soil moisture sensor. In [32] a smartphone App is designed which enables the Wi-Fi connection of the smartphone creating an access point to a router node. This node is connected through the Zigbee protocol to a gateway that controls the irrigation pump. In [33], authors presented wireless sensor network to monitor the soil properties of the farm by using a 6LoWPAN. 4G cellular technology is also considered as a good choice in underground wireless sensor networks [34]. Common IoT applications which use RFID are security, medical, smart shopping and smart agriculture. RFID based smart irrigation system was presented in [35] which used soil moisture and soil temperature sensors for controlling valve of irrigation pump. An information collected by these sensors is transmitted to the cloud based on RFID wireless communication protocols, with the help of which user can control a water pump.

\section{Precision Agriculture Challenges}

Precision agriculture is in use from last few decades to increase crop productivity by keeping low cost and less human intervention. But still very limited farmers have adopted these techniques due to several challenges. As Precision agriculture relies on different sensors which have certain limitations like deployment, development cost and maintenance cost. So this hardware cost is main challenge in precision agriculture. Atmospheric disturbances also cause interference in wireless communication channels which can further interrupt communication between wireless nodes and cloud. In Precision agriculture systems large amounts of data get generated which need storage and data analysis. New facilities and software platforms are in demand for management of data. Literacy and connectivity are another important factors that affects the adoption of smart farming. The main challenge that is acting as an obstruction in growth of precision agriculture is interoperability.

\section{Conclusion and Future scope}

Precision agriculture with involvement of technologies like IoT and wireless sensor networks helps to increase yield and productivity with resource optimization. Most of the research articles in this field indicates that precision agriculture is about sustainability and decision support systems. Precision agriculture includes multiple parameters like temperature, soil moisture, light intensity, humidity, soil nutrients, etc. Many challenges are also involved in the system developed for precision agriculture. New software platforms and advanced technologies can further help in development of smart agriculture.

\section{References}

A. C. Jose, "Improving home automation security; in- tegrating device fingerprinting into smart home," IEEE Access, vol. 4, pp. 5776-5787, 2016.

A. Gluhak, S. Krco, M. Nati. "A survey of facilities for the experimental internet of things research". IEEE Communications Magazine, 49(11), pp.58-67, 2011.

1. Agrawal, S., Vieira, D., 2013, “A survey on Internet of Things. J. Network Computing. App1 (2), pp. 78-95.

2. Al-Sarawi, S.; Anbar, M.; Alieyan, K.; Alzubaidi, M. Internet of Things (IoT) communication protocols. In Proceedings of the 2017 8th International Conference on Information Technology (ICIT), Amman, Jordan, 17-18 May 2017; pp. 685-690.

3. Atzori, L., Iera, A., Morabito, G., 2010. "The Internet of Things: A survey". Computer Networks 54 (15), pp. 2787-2805.

4. Balducci, F., Impedovo, D., Pirlo, G., 2018. "Machine learning applications on agricultural datasets for smart farm enhancement". Machines 6 (3), 38.

5. Bright Keswani,Ambarish G. Mohapatra,Amarjeet Mohanty,Ashish Khanna,Joel J. P. C. Rodrigues,Deepak Gupta, Victor Hugo C. de Albuquerque, "Adapting weather conditions based IoT enabled smart irrigation technique in precision agriculture mechanisms ", Neural Computing and Applications https://doi.org/10.1007/s00521-018-3737-1.12.3456789().,-volV)(0123456789().,volV)

6. Chikankar, P.B.; Mehetre, D.; Das, S. An automatic irrigation system using ZigBee in wireless sensor network. In Proceedings of the 2015 International Conference on Pervasive Computing (ICPC), Pune, India, 8-10 January 2015; pp. 1-5.

7. Farzad Kiani, Amir Seyyedabbasi. "Wireless Sensor Network and Internet of Things in Precision Agriculture “. International Journal of Advanced Computer Science and Applications, Vol. 9, No. 6, 2018, pp. 99-103.

8. G. Mendez, M.A. Yunus and S.C. Mukhopadhyay. "A WiFi-based smart wireless sensor network for monitoring an agricultural environment". IEEE Conference in Instrumentation and Measurement Technology Conference (I2MTC), pp. 2640-2645, 2012.

9. G. Mendez, M.A. Yunus and S.C. Mukhopadhyay. "A WiFi-based smart wireless sensor network for monitoring an agricultural environment". IEEE Conference in Instrumentation and Measurement Technology Conference (I2MTC), pp. 2640-2645, 2012.

10. https://en.wikipedia.org/wiki/Economic_survey_of_India

11. https://shodhganga.inflibnet.ac.in/bitstream/10603/50407/14/14_chapter\%205.pdf 
12. Indu, G., Reddy, S.R.N., 'Innovative GSM bluetooth based remote controlled embedded system for irrigation', Int. J. Comput. Appl., 2012, 47, (13), pp. 1-7.

13. J. Wolfert, C. Srensen, and D. Goense, "A future internet collaboration platform for safe and healthy food from farm to fork," in 2014 Annual SRII. IEEE, 2014, pp. 266-273

14. Joaquín Gutiérrez, Juan Francisco Villa-Medina, Aracely López-Guzmán, and Miguel Ángel PortaGándara. "Smartphone Irrigation Sensor “, IEEE Sensors Journal Sensors-12234-2015, pp. 1-7.

15. K. Shinghal and S. Neelam. "Wireless Sensor Networks in Agriculture: For Potato Farming". Available at SSRN: https://ssrn.com/abstract=3041375, 2017.

16. L. Atzori, A. Iera and G. Morabito, "The Internet of Things: A survey,” Computer Network, 54(15), pp. 2787-2805, 2010

17. Liao, M.S., Chen, S.F., Chou, C.Y.”On precisely relating the growth of Phalaenopsis leaves to greenhouse environmental factors by using an IoT-based monitoring system", Computers and Electronics in Agriculture,136, 2017 ,pp. 125-139.

18. M. Ibrahim, M. Rawidean, M. Kassim and A.N. Harun. "IoT in precision agriculture applications using wireless moisture sensor network". IEEE Conference on Open Systems, pp.24-29, 2016

19. Mahir, D., Semih, O.: 'A wireless application of drip irrigation automation supported by soil moisture sensors', Sci. Res. Essays, 2011, 6, (7), pp. 1573-1582

20. Majone, B., F. Viani, E. Filippi, A. Bellin, A. Massa, G. Toller, F. Robol, and M. Salucci. 2013. "WSN Deployment for Monitoring Soil Moisture Dynamics at the Field Scale, International Conference on Four Decades of Progress in Monitoring and Modeling ofProcesses in Soil Plant Atmosphere System: Applications and Challenges." In Procedia Environmental Sciences, 19, Naples, Italy, 426-435.

21. Meeradevi, M. A. Supreetha, Monica R. Mundada and J. N. Pooja, "Design of a Smart Water-Saving Irrigation System for Agriculture Based on a Wireless Sensor Network for Better Crop Yield “, A. Kumar and S. Mozar (eds.), ICCCE 2018, Lecture Notes in Electrical Engineering 500,https://doi.org/10.1007/978-981-13-0212-1_11,pp. 93-104.

22. Mei-Hui Liang, Yao-Feng He, Li-Jun Chen, Shang-Feng Du, "Greenhouse Environment dynamic Monitoring system based on WIFI “, IFAC conference Papers On Line 51-17 (2018) pp.736-740.

23. N. Zhang, M. Wang, and N. Wang, "Precision agricul- ture* a worldwide overview," Computers and Electronics in Agriculture, vol. 36, pp. 113-132, 2002

24. N-USha, T.M. Conditions in Agriculture through WiFi using Raspberry PI. Int. J. Eng. 2017, 3, 611.

25. Paventhan, A.; Allu, S.K.; Barve, S.; Gayathri, V.; Ram, N.M. Soil property monitoring using 6lowpan-enabled wireless sensor networks. In Proceedings of the Agro-Informatics and Precision Agriculture, Hyderabad, India, 1-3 August 2012.

26. Sabrine Khriji, Dhouha El Houssaini, Mohamed Wassim Jmal, Christian Viehweger, Mohamed Abid, Olfa Kanoun,." Precision irrigation based on wireless sensor network ", IET Sci. Meas. Technol., 2014, Vol. 8, Iss. 3, pp. 98-106.

27. Tanaka, K.; Murase, M.; Naito, K. Prototype implementation of BLE based automated data collection scheme in agricultural measurement system. In Proceedings of the 2018 15th IEEE Annual Consumer Communications \& Networking Conference (CCNC), Las Vegas, NV, USA, 12-15 January 2018; pp. 1-2.

28. Uferah Shafi 1 , Rafia Mumtaz 1,*, José García-Nieto 2, Syed Ali Hassan 1 , Syed Ali Raza Zaidi 3 and Naveed Iqbal 1 , Sensors 2019, 19, 3796; doi:10.3390/s19173796 pp. 1-25.

29. Wasson, T.; Choudhury, T.; Sharma, S.; Kumar, P. Integration of RFID and sensor in agriculture using IOT. In Proceedings of the 2017 International Conference On Smart Technologies For Smart Nation (SmartTechCon), Bangalore, India, 17-19 August 2017; pp. 217-222 .

30. www.livemint.com

31. X. Jianfa. "An environment monitoring system for precise agriculture based on wireless sensor networks". IEEE International Conference Mobile Ad-hoc and Sensor Networks (MSN), pp. 28-35, 2011

32. Zhang, X.; Andreyev, A.; Zumpf, C.; Negri, M.C.; Guha, S.; Ghosh, M. Thoreau: A subterranean wireless sensing network for agriculture and the environment. In Proceedings of the 2017 IEEE Conference on Computer Communications Workshops (INFOCOM WKSHPS), Atlanta, GA, USA, 1-4 May 2017; pp. 78-84.

33. Zhou, Y.; Yang, X.; Guo, X.; Zhou, M.; Wang, L. A design of greenhouse monitoring \& control system based on ZigBee wireless sensor network. In Proceedings of the 2007 International Conference on Wireless Communications, Networking and Mobile Computing, Shanghai, China, 21-25 September 2007; pp. 2563-2567. 